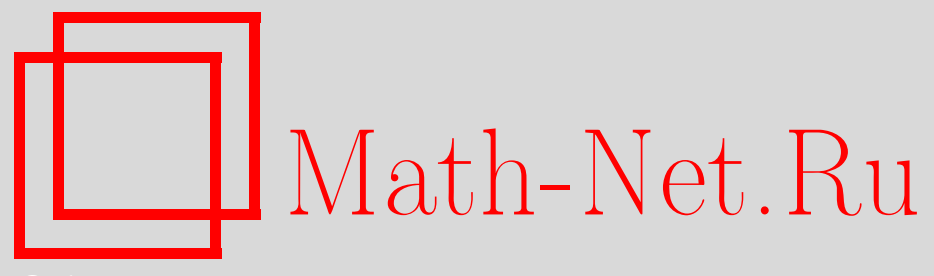

Р. Х. Каримов, Л. М. Кожевникова, Стабилизация решений квазилинейных параболических уравнений второго порядка в областях с некомпактными границами, $M a-$ тем. сб., 2010, том 201, номер 9, 3-26

DOI: https://doi.org/10.4213/sm7602

Использование Общероссийского математического портала Math-Net.Ru подразумевает, что вы прочитали и согласны с пользовательским соглашением http://www . mathnet.ru/rus/agreement

Параметры загрузки:

IP: 34.229 .45 .116

26 апреля 2023 г., 13:26:26

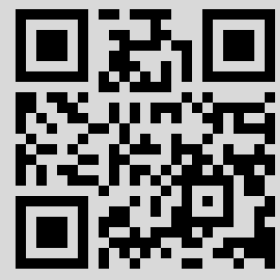




\author{
Р. Х. Каримов, Л. М. Кожевникова
}

\title{
Стабилизация решений квазилинейных параболических уравнений второго порядка в областях с некомпактными границами
}

Для квазилинейных параболических уравнений второго порядка в цилиндрической области $D=(0, \infty) \times \Omega$ рассматривается первая смешанная задача с однородным краевым условием Дирихле и финитной начальной функцией. Установлены оценки сверху, характеризующие зависимость скорости убывания решений при $t \rightarrow \infty$ от геометрии неограниченной области $\Omega \subset \mathbb{R}_{n}, n \geqslant 2$.

Библиография: 18 названий.

Ключевые слова: первая смешанная задача, квазилинейные параболические уравнения, неограниченная область, стабилизация решения, геометрическая характеристика.

\section{Введение}

Пусть $\Omega$ - неограниченная область пространства $\mathbb{R}_{n}=\left\{\mathbf{x}=\left(x_{1}, x_{2}, \ldots, x_{n}\right)\right\}$, $n \geqslant 2$. В цилиндрической области $D=\{t>0\} \times \Omega$ для квазилинейного параболического уравнения второго порядка рассматривается первая смешанная задача

$$
\begin{gathered}
u_{t}=\sum_{\alpha=1}^{n}\left(a_{\alpha}(t, \mathbf{x}, \nabla u)\right)_{x_{\alpha}}-a(t, \mathbf{x}, u), \quad(t, \mathbf{x}) \in D ; \\
\left.u(t, \mathbf{x})\right|_{S}=0, \quad S=\{t>0\} \times \partial \Omega ; \\
u(0, \mathbf{x})=\varphi(\mathbf{x}), \quad \varphi(\mathbf{x}) \in L_{2}(\Omega) .
\end{gathered}
$$

Предполагается, что функции, входящие в уравнение (0.1), удовлетворяют следующим требованиям. Функции $a_{\alpha}(t, \mathbf{x}, \xi), \alpha=\overline{1, n}$, измеримы по $(t, \mathbf{x}) \in D$ и для всех $\xi, \eta \in \mathbb{R}_{n}$ при п.в. $(t, \mathbf{x}) \in D$ подчиняются условиям

$$
\begin{gathered}
\sum_{\alpha=1}^{n}\left(a_{\alpha}(t, \mathbf{x}, \xi)-a_{\alpha}(t, \mathbf{x}, \eta)\right)\left(\xi_{\alpha}-\eta_{\alpha}\right) \geqslant \bar{a}|\xi-\eta|^{m+1}, \quad m \geqslant 1 ; \\
|\mathbf{a}(t, \mathbf{x}, \xi)-\mathbf{a}(t, \mathbf{x}, \eta)| \leqslant \widehat{a}|\xi-\eta|(|\xi|+|\eta|)^{m-1}, \quad \mathbf{a}=\left(a_{1}, \ldots, a_{n}\right) \\
a_{\alpha}(t, \mathbf{x}, \mathbf{0})=0, \quad \alpha=\overline{1, n} .
\end{gathered}
$$

Работа выполнена при поддержке Российского фонда фундаментальных исследований (грант № 09-01-00440-а). 
Функция $a(t, \mathbf{x}, s)$ измерима по $(t, \mathbf{x}) \in D$ и для всех $s, r \in \mathbb{R}$ при п.в. $(t, \mathbf{x}) \in D$ подчиняется условиям

$$
\begin{gathered}
(a(t, \mathbf{x}, s)-a(t, \mathbf{x}, r))(s-r) \geqslant 0 ; \\
|a(t, \mathbf{x}, s)-a(t, \mathbf{x}, r)| \leqslant \widetilde{a}|s-r|(|s|+|r|)^{q_{*}-1}, \quad 1 \leqslant q_{*} \leqslant q, \quad q=m+\frac{2(m+1)}{n} ; \\
a(t, \mathbf{x}, 0)=0 .
\end{gathered}
$$

Здесь $\bar{a}, \widehat{a}, \widetilde{a}-$ положительные числа.

Линейное параболическое уравнение

$$
u_{t}=\sum_{\alpha, \beta=1}^{n}\left(a_{\alpha \beta}(t, \mathbf{x}) u_{x_{\beta}}\right)_{x_{\alpha}}
$$

является примером уравнения (0.1) с функциями

$$
a_{\alpha}(t, \mathbf{x}, \nabla u)=\sum_{\beta=1}^{n} a_{\alpha \beta}(t, \mathbf{x}) u_{x_{\beta}}, \quad \alpha=\overline{1, n}, \quad a(t, \mathbf{x}, u)=0 \quad \text { при } m=1 .
$$

Работа посвящена исследованию зависимости скорости стабилизации при $t \rightarrow \infty$ от геометрии неограниченной области $\Omega$ решения задачи $(0.1)-(0.3)$ с финитной начальной функцией $\varphi(\mathbf{x})$.

А.К. Гущин положил начало изучению скорости убывания при больших значениях времени решений смешанных задач с начальной функцией, ограниченной в одной из $L_{p}$-норм, для параболических уравнений в неограниченных областях. В широком классе неограниченных областей в терминах простой геометрической характеристики $v(r)=\operatorname{mes} \Omega(r), \Omega(r)=\{\mathbf{x} \in \Omega|| \mathbf{x} \mid<r\}$, в работах [1], [2] А. К. Гущиным установлены точные оценки решений второй смешанной задачи для уравнения $\left(0.1^{\prime}\right)$.

Исследованию поведения решений смешанных задач для линейных параболических уравнений второго и высокого порядков при $t \rightarrow \infty$ посвящены работы А. В. Лежнева, В. И. Ушакова, Ф. Х. Мукминова, Л. М. Кожевниковой, И. М. Биккулова и др. Обзоры соответствующих результатов можно найти в [3] и [4]. Здесь остановимся лишь на некоторых результатах для нелинейных параболических уравнений.

А. Ф. Тедеев (см. [5]) получил оценку сверху $L_{2}$-нормы решения первой смешанной задачи для параболического квазилинейного уравнения высокого порядка в дивергентной форме

$$
u_{t}+(-1)^{p} \sum_{|\bar{\alpha}|=p} D^{\bar{\alpha}} A_{\bar{\alpha}}\left(t, \mathbf{x}, u, D u, \ldots, D^{p} u\right)=0, \quad p \geqslant 1,
$$

где

$$
D^{\bar{\alpha}}=\frac{\partial^{|\bar{\alpha}|}}{\partial x_{1}^{\alpha_{1}} \cdots \partial x_{n}^{\alpha_{n}}}, \quad \bar{\alpha}=\left(\alpha_{1}, \ldots, \alpha_{n}\right), \quad|\bar{\alpha}|=\alpha_{1}+\cdots+\alpha_{n} .
$$


Здесь $A_{\bar{\alpha}}(t, \mathbf{x}, \xi)$ - каратеодориевы функции, удовлетворяющие условиям

$$
\begin{gathered}
\sum_{|\bar{\alpha}|=p} A_{\bar{\alpha}}(t, \mathbf{x}, \xi) \xi_{\bar{\alpha}}^{p} \geqslant a_{1} \sum_{|\bar{\alpha}|=p}\left|\xi_{\bar{\alpha}}^{p}\right|^{m+1}, \quad m \geqslant 1, \\
\sum_{|\bar{\alpha}|=p}\left|A_{\bar{\alpha}}(t, \mathbf{x}, \xi)\right| \leqslant a_{2} \sum_{|\bar{\alpha}|=p}\left|\xi_{\bar{\alpha}}^{p}\right|^{m}
\end{gathered}
$$

для любого вектора $\xi=\left(\xi^{0}, \xi^{1}, \ldots, \xi^{p}\right), \xi^{i}=\left(\xi_{\bar{\alpha}}^{i}\right),|\bar{\alpha}|=i, a_{1}, a_{2}$ - положительные постоянные. Для решения (ограниченного при $m>1$ ) уравнения $(0.10)$ с граничным условием

$$
\left.D^{\bar{\alpha}} u(t, \mathbf{x})\right|_{S}=0, \quad|\bar{\alpha}| \leqslant p-1,
$$

с финитной начальной функцией $\varphi(\mathbf{x}) \in L_{2}(\Omega)$ для $t>0$ установлена оценка

$$
\begin{aligned}
& \|u(t)\|_{L_{2}(\Omega \backslash \Omega(r))} \leqslant C_{1} \exp \left\{-b_{1}\left[\frac{\left(r-r_{0}\right)^{p(m+1)}}{t}\right]^{1 /(p(m+1)-1)}\right\}\|\varphi\|_{L_{2}(\Omega)}, \\
& r \geqslant r_{0}>0 \text {. }
\end{aligned}
$$

На основе неравенства $(0.12)$ для $m=1$ при достаточно больших $t$ получена оценка

$$
\|u(t)\|_{L_{2}(\Omega)} \leqslant C_{2} \exp \left\{-b_{2}\left[\frac{r^{2 p}(t)}{t}\right]^{1 /(2 p-1)}\right\}\|\varphi\|_{L_{2}(\Omega)} .
$$

Здесь функция $r(t), t>0$, определяется из равенства $\lambda^{2 p-1}(r) t^{2}=r^{2} ; \lambda(r)$, $r>0,-$ первое собственное значение задачи Дирихле для оператора $-\Delta$ в $\Omega(r)$. Ранее аналогичный результат для линейного параболического уравнения высокого порядка был получен Ф. Х. Мукминовым (см. [6]).

При $m>1$ в работе [5] показано, что для ограниченного решения задачи $(0.10),(0.11),(0.3)$ в области вращения $\Omega\left(f_{a}\right)=\left\{\left(x_{1}, \mathbf{x}^{\prime}\right) \in \mathbb{R}_{n} \mid x_{1}>0\right.$, $\left.\left|\mathbf{x}^{\prime}\right|<x_{1}^{a}\right\}, a>0$, при достаточно больших $t$ имеет место неравенство

$$
\|u(t)\|_{L_{2}(\Omega)} \leqslant C_{3} t^{-\alpha}, \quad C_{3}>0 .
$$

При этом положительный показатель $\alpha$ определяется постоянными $n, a, m, p$ (точное значение не приводится).

В [7] А. Ф. Тедеевым исследовалась допустимая скорость стабилизации решения первой смешанной задачи для квазилинейного параболического уравнения высокого порядка; приведем результаты этой работы для уравнения

$$
u_{t}+(-1)^{p} \sum_{|\bar{\alpha}|=p} D^{\bar{\alpha}}\left(\left|D^{p} u\right|^{m-1} D^{\bar{\alpha}} u\right)+|u|^{q_{*}-1} u=0, \quad p \geqslant 1, \quad m \geqslant 1, \quad q_{*} \geqslant m .
$$

Заметим, что при $p=1$ и соответствующем значении $q_{*}$ уравнение $(0.13)$ является примером уравнения (0.1). В [7] для решения задачи $(0.13),(0.11),(0.3)$ при $t>0$ доказана оценка

$$
\|u(t)\|_{L_{2}(\Omega)} \geqslant\|\varphi\|_{L_{2}(\Omega)}\left(C_{4}(\varphi) t+1\right)^{-1 /(m-1)}, \quad m>1 .
$$


Оценка снизу для решения задачи Коши в случае уравнения

$$
u_{t}+(-1)^{p} \sum_{|\bar{\alpha}|=p} D^{\bar{\alpha}}\left(\left|D^{p} u\right|^{m-1} D^{\bar{\alpha}} u\right)=0, \quad p \geqslant 1, \quad m \geqslant 1,
$$

при $p=1$ получена в работе [8].

Кроме того, в работе [7] для решения задачи $(0.15),(0.11),(0.3)$ при $t>0$ установлены оценки сверху

$$
\begin{array}{ll}
\|u(t)\|_{L_{\infty}(\Omega)} \leqslant C_{6} t^{-\beta}, & n<p(m+1), \\
\|u(t)\|_{L_{r}(\Omega)} \leqslant C_{5} t^{-(r-2) \beta / r}, & n \geqslant p(m+1), \quad r \geqslant 2, \\
\quad \beta=n\{n(m-1)+2(m+1) p\}^{-1} .
\end{array}
$$

Следует отметить, что последняя оценка является тривиальной при $r=2$. Поэтому можно ожидать, что она не является точной и при других значениях $r$.

В работах [9], [10] рассматривались вторая и третья смешанные задачи для квазилинейных параболических уравнений второго порядка и получены точные оценки скорости стабилизации решений при $t \rightarrow \infty$. Отметим еще, что большое число работ посвящено исследованию скорости убывания решения задачи Коши для нелинейных параболических уравнений (см., например, [11] и имеющиеся там ссылки). В ряде работ изучается решение задачи Коши для параболического уравнения с источником. Хорошо известно, что такая задача может не иметь глобального по времени решения вне зависимости от свойств начальной функции. Поэтому важным является вопрос об условиях на параметры уравнения, при которых существует глобальное по времени решение. Обзор результатов по данному направлению исследований можно найти в работе [12].

Основная идея из работы Ф.Х. Мукминова [13] получения оценки сверху решения первой смешанной задачи для линейного параболического уравнения в неограниченной области $\Omega$ заключается в следующем. Сначала устанавливается оценка убывания решения по мере удаления на бесконечность области $\Omega$. Затем для фиксированного значения $t$ выбирается ограниченная часть области $\Omega$, за пределами которой решение "пренебрежимо мало", и в этой ограниченной части устанавливается оценка убывания решения по времени. В настоящей работе такой же метод применяется для квазилинейного уравнения.

Для изучения убывания решения задачи (0.1)-(0.3) при $|\mathbf{x}| \rightarrow \infty$ будем пользоваться понятием $\lambda$-разбиения области, которое позволяет получать точные оценки решений краевых задач для линейных эллиптических и параболических уравнений. Это понятие можно считать обобщением понятия $\lambda$-последовательности, введенного ранее в работах [4], [14]-[16] для областей, расположенных вдоль выделенной оси. Следует отметить, что в работе [17] О.А. Олейник и Г. А. Иосифьян по существу использовали прототип такой последовательности для системы уравнений теории упругости.

В настоящей работе, адаптируя понятие $\lambda$-разбиения на квазилинейный оператор, устанавливаем равномерные по времени оценки сверху для уравнения (0.1) в широком классе областей с некомпактными границами. 
Будем предполагать, что неограниченная область $\Omega \subset \mathbb{R}_{n}$ представлена в виде объединения $\Omega=\bigcup_{N=0}^{\infty} \Omega^{(N)}$ последовательности вложенных $\Omega^{(N)} \subset \Omega^{(N+1)}$ ограниченных областей, удовлетворяющих следующим требованиям. Дополнения $\Omega_{(N-1)}^{(N)}=\Omega^{(N)} \backslash \overline{\Omega^{(N-1)}}$ распадаются на конечное число связных компонент $\omega_{i}^{(N)}, i=\overline{1, p^{(N)}}$ :

$$
\Omega_{(N-1)}^{(N)}=\bigcup_{i=1}^{p^{(N)}} \omega_{i}^{(N)}, \quad N=\overline{1, \infty} .
$$

Пересечения $\left(\partial \Omega^{(N)}\right) \cap \Omega=S^{(N)}, N=\overline{0, \infty}$, представляют собой конечное число липшицевых гиперповерхностей $S_{i}^{(N)}=\partial \omega_{i}^{(N)} \cap S^{(N)}\left(S_{i}^{(N)} \neq 0\right.$ могут быть несвязными), $i=\overline{1, p^{(N)}}, N=\overline{1, \infty}$.

Для множества $Q \subset \Omega$ введем обозначение

$$
\lambda[Q]=\inf \left\{\|\nabla g\|_{L_{m+1}(Q)} \mid g(\mathbf{x}) \in C_{0}^{\infty}(\Omega),\|g\|_{L_{m+1}(Q)}^{m+1}=1\right\} .
$$

Определим векторы $t^{(N)}=\left(t_{1}^{(N)}, \ldots, t_{p^{(N)}}^{(N)}\right)$ и $\lambda^{(N)}=\left(\lambda_{1}^{(N)}, \ldots, \lambda_{p^{(N)}}^{(N)}\right)$ формулами

$$
t_{i}^{(N)}=\operatorname{dist}\left(S_{i}^{(N)}, \widetilde{S}_{i}^{(N-1)}\right),
$$

где $\widetilde{S}_{i}^{(N-1)}=\partial \omega_{i}^{(N)} \cap S^{(N-1)} \neq 0, \lambda_{i}^{(N)}=\lambda\left[\omega_{i}^{(N)}\right], i=\overline{1, p^{(N)}}, N=\overline{1, \infty}$. Будем предполагать, что существует число $\theta>0$ такое, что выполняются неравенства

$$
1 \leqslant \theta \lambda_{i}^{(N)}\left(t_{i}^{(N)}\right)^{m+1}, \quad i=\overline{1, p^{(N)}}, \quad N=\overline{1, \infty} .
$$

Описанное выше представление $\Omega=\bigcup_{N=0}^{\infty} \Omega^{(N)}$ при выполнении неравенств (0.17) будем называть $\lambda$-разбиением области.

Для неограниченных областей, расположенных вдоль выделенной оси $O x_{1}$ (область $\Omega$ лежит в полупространстве $\mathbb{R}_{n}^{+}=\left\{\mathbf{x} \in \mathbb{R}_{n} \mid x_{1}>0\right\}$, сечение $\gamma_{r}=\left\{\mathbf{x} \in \Omega \mid x_{1}=r\right\}$ непусто и ограничено при любом $\left.r>0\right)$, множества $\Omega^{(N)}=\Omega^{z_{N}}=\left\{\mathbf{x} \in \Omega \mid 0<x_{1}<z_{N}\right\}$ можно определить с помощью неограниченной возрастающей последовательности положительных чисел $\left\{z_{N}\right\}_{N=0}^{\infty}$. При этом последовательность $\left\{z_{N}\right\}_{N=0}^{\infty}$ называется $\lambda$-последовательностъю, а условие (0.17) для разбиения $\Omega=\bigcup_{N=0}^{\infty} \Omega^{z_{N}}$ принимает вид

$$
1 \leqslant \theta \lambda\left(z_{N-1}, z_{N}\right) \Delta_{N}^{m+1}, \Delta_{N}=z_{N}-z_{N-1}, \quad N=\overline{1, \infty} .
$$

Здесь и ниже используется обозначение $\lambda\left(r_{1}, r_{2}\right)=\lambda\left[\Omega_{r_{1}}^{r_{2}}\right]$, где $\Omega_{r_{1}}^{r_{2}}=\{\mathbf{x} \in \Omega \mid$ $\left.r_{1}<x_{1}<r_{2}\right\}$, значения параметров $r_{1}=0$ и $r_{2}=\infty$ могут быть опущены.

Приведем необходимое и достаточное условие существования $\lambda$-последовательности $\left\{z_{N}\right\}_{N=0}^{\infty}$ (см. следствие к утверждению 2):

$$
\text { для любого } r_{1}>0 \text { найдется } r_{2}>r_{1} \text { такое, что } \lambda\left(r_{1}, r_{2}\right)>0 \text {. }
$$

Предполагается, что начальная функция имеет ограниченный носитель, так что

$$
\operatorname{supp} \varphi \subset \Omega^{(0)}
$$


Будем считать, что выполнено условие

$$
\lambda^{(0)}=\lambda\left[\Omega^{(0)}\right]>0
$$

(этого можно добиться сдвигом нумерации $\widetilde{N}=N+k$ ).

ТЕОРема 1. Пусть для области $\Omega$ существует $\lambda$-разбиение $\Omega=\bigcup_{N=0}^{\infty} \Omega^{(N)}$ $u$ выполнены условия (0.4)-(0.9), (0.20). Тогда найдутся положительные числа $\varkappa_{m}(\bar{a}, \widehat{a}, \theta), M(m, \bar{a}, \widehat{a}, \theta)$ такие, что обобщенное решение $u(t, \mathbf{x})$ задачи (0.1)-(0.3) при всех $t \geqslant 0, N \geqslant 0$ удовлетворяет оченке

$$
\|u(t)\|_{L_{2}\left(\Omega \backslash \Omega^{(N)}\right)} \leqslant M e^{-\varkappa_{m} N}\|\varphi\|_{L_{2}(\Omega)} .
$$

В случае линейного уравнения $\left(0.1^{\prime}\right)$ оценка $(0.22)$ является точной в том смысле, что если в ней заменить $\varkappa_{m}$ на любую растущую на бесконечности последовательность $\varkappa_{m}(N)$, то она становится неверной (для линейного эллиптического уравнения аналогичный факт установлен в [15; теорема 3]). Сформулируем теперь результат об убывании решения задачи (0.1)-(0.3) при $t \rightarrow \infty$.

Определим последовательность

$$
\begin{gathered}
F_{m}(N)=\frac{1}{\inf \left\{\|\nabla g\|_{L_{m+1}\left(\Omega^{(N)}\right)} \mid g(\mathbf{x}) \in C_{0}^{\infty}(\Omega),\|g\|_{L_{2}\left(\Omega^{(N)}\right)}=1\right\}}, \\
m \geqslant 1, \quad N=\overline{0, \infty}
\end{gathered}
$$

Пусть $N_{m}(t), m>1, N_{1}(t)$ - произвольные неотрицательные функции, удовлетворяющие соответственно неравенствам

$$
\begin{gathered}
F_{m}^{m+1}\left(N_{m}(t)\right) \exp \left(\varkappa_{m}(m-1) N_{m}(t)\right) \geqslant t, \quad m>1, \quad t>0 ; \\
N_{1}(t) F_{1}^{2}\left(N_{1}(t)\right) \leqslant t, \quad t \geqslant 0 .
\end{gathered}
$$

Например, можно положить

$$
\begin{gathered}
N_{m}(t)=\min \left\{N \in \overline{0, \infty} \mid F_{m}^{m+1}(N) \exp \left(\varkappa_{m}(m-1) N\right) \geqslant t\right\}, \quad m>1, \quad t \geqslant 0 ; \\
N_{1}(t)=\max \left\{N \in \overline{0, \infty} \mid N F_{1}^{2}(N) \leqslant t\right\}, \quad t \geqslant 0 .
\end{gathered}
$$

ТЕОРема 2. Пусть для области $\Omega$ существует $\lambda$-разбиение $\Omega=\bigcup_{N=0}^{\infty} \Omega^{(N)}$ и выполнены условия (0.4)-(0.9), (0.20), (0.21). Тогда найдутся положительнъе числа $\varkappa^{*}(\theta, \bar{a}, \widehat{a}), M_{m}(\theta, \bar{a}, \widehat{a},\|\varphi\|)$ такие, что для решения $u(t, \mathbf{x})$ задачи (0.1)-(0.3) справедливы оценки:

$$
\begin{array}{ll}
\text { nри } m>1 \quad\|u(t)\|_{L_{2}(\Omega)} \leqslant M_{m} t^{-1 /(m-1)} F_{m}^{(m+1) /(m-1)}\left(N_{m}(t)\right), \quad t>0 ; \\
\text { nри } m=1 \quad\|u(t)\|_{L_{2}(\Omega)} \leqslant M_{1} \exp \left(-\varkappa^{*} N_{1}(t)\right), \quad t \geqslant 0 .
\end{array}
$$

Следует отметить, что точность оценки (0.27) для линейного уравнения $\left(0.1^{\prime}\right)$ в широком классе областей вращения установлена в [4; теорема 2].

Заметим, что практически всегда можно устроить разбиение с выполнением следующего условия: существует положительная константа $b$ такая, что

$$
F_{m}(N) \leqslant C \exp (b N), \quad N \geqslant 0 .
$$


ПредЛОЖЕНИЕ 1. Пусть $\lambda$-разбиение $\Omega=\bigcup_{N=0}^{\infty} \Omega^{(N)}$ удовлетворяет условиям: существуют постоянные $a \geqslant 1, c_{1}, c_{2}>0$ такие, что справедливь неравенства

$$
\begin{gathered}
\operatorname{mes} \Omega^{(N)} \leqslant c_{1} a^{N n}, \\
t_{i}^{(N)} \leqslant c_{2} a^{N}, \quad i=\overline{1, p^{(N)}}, \quad N=\overline{1, \infty} .
\end{gathered}
$$

Тогда существует положительное число ь такое, что верна оценка (0.28).

Если выполнено условие (0.28), то можно положить

$$
\begin{aligned}
& \exp \left(N_{m}(t)\right)=t^{1 /\left(b(m+1)+\varkappa_{m}(m-1)\right)}, \quad m>1, \\
& \exp \left(N_{1}(t)\right)=t^{1 /(2 b+\varepsilon)}, \quad \varepsilon \in(0,1), \quad t>0,
\end{aligned}
$$

и оценки (0.26), (0.27) принимают вид

$$
\begin{gathered}
\|u(t)\|_{L_{2}(\Omega)} \leqslant M_{m} t^{-\varkappa_{m} /\left(b(m+1)+\varkappa_{m}(m-1)\right)}, \quad m>1, \quad t>0, \\
\|u(t)\|_{L_{2}(\Omega)} \leqslant M_{1} t^{-\varkappa_{1} /(2 b+\varepsilon)}, \quad m=1, \quad t>0 .
\end{gathered}
$$

Если же выполнено условие

$$
\lim _{N \rightarrow \infty} \frac{\ln F_{m}(N)}{N}=0
$$

то можно выбрать

$$
\exp \left(N_{m}(t)\right)=t^{1 /\left(\varkappa_{m}(m-1)\right)}, \quad m>1, \quad t>0,
$$

и оценка (0.26) принимает вид

$$
\|u(t)\|_{L_{2}(\Omega)} \leqslant M_{m} t^{-(1-\varepsilon) /(m-1)}, \quad m>1, \quad \varepsilon \in(0,1), \quad t>0 .
$$

Выбор функции $N_{m}(t)$ формулой (0.34) является оптимальным, поскольку оценка (0.35) имеет показатель степени, близкий к показателю $1 /(m-1)$ оценки снизу (0.14).

В качестве примера рассмотрим полупространство $\mathbb{R}_{n}^{+}$. Определим множества $\Omega^{(N)}$ как $\left\{\mathbf{x} \in \mathbb{R}_{n}^{+}|| \mathbf{x} \mid<2^{N}\right\}, N=\overline{0, \infty}$. При этом справедливы соотношения

$$
t^{(N)}=2^{N-1}, \quad \operatorname{mes} \Omega^{(N)}=c_{1} 2^{N n}, \quad \lambda^{(N)}=c_{2} 2^{-(m+1) N}, \quad N=\overline{1, \infty},
$$

и выполняются неравенства (0.17). Поэтому такие множества $\Omega^{(N)}$ осуществляют $\lambda$-разбиение полупространства $\mathbb{R}_{n}^{+}$. Таким образом, справедливы условия $(0.29),(0.30)$ и имеет место оценка $(0.31)$.

Рассмотрим область вращения

$$
\Omega(f)=\left\{\left(x_{1}, \mathbf{x}^{\prime}\right) \in \mathbb{R}_{n}\left|x_{1}>0,\right| \mathbf{x}^{\prime} \mid<f\left(x_{1}\right)\right\}
$$

с положительной функцией $f\left(x_{1}\right)<\infty$. От функции $f$ требуется только, чтобы множество $\Omega(f)$ было областью. 
Для областей вращения вида (0.36) выразим оценки (0.22), (0.27) через функцию $f(x)$, с этой целью введем понятие П-последовательности.

Неограниченную возрастающую последовательность положительных чисел $\left\{z_{N}\right\}_{N=0}^{\infty}$ определим равенствами

$$
z_{0}=1, \quad z_{N}=\sup \left\{r>z_{N-1} \mid \inf _{\left[z_{N-1}, r\right)} f(x) \geqslant r-z_{N-1}\right\}, \quad N=\overline{1, \infty}
$$

и назовем П-последовательностъю функции $f(x)$. Установлено, что П-последовательность является $\lambda$-последовательностью для области $\Omega(f)$ (см. утверждение 3).

Несложным следствием теоремы 1 для областей вращения является следующая оценка (см. утверждение 4):

$$
\|u(t)\|_{L_{2}\left(\Omega_{r}\right)} \leqslant \widetilde{M} \exp \left(-\varkappa_{m} \int_{1}^{r} \frac{d x}{f(x)}\right)\|\varphi\|_{L_{2}(\Omega)}, \quad t \geqslant 0, \quad r \geqslant 1 .
$$

Следует отметить, что при $r<t^{1 /(m+1)}$ и достаточно больших $t$ для областей вращения, удовлетворяющих условию $\int_{1}^{\infty} \frac{d x}{f(x)}=\infty$, оценка (0.38) дает более высокий порядок убывания, чем оценка (0.12) при $p=1$.

Предположим, далее, что функция $f$ удовлетворяет условию

$$
\lim _{r \rightarrow \infty} \frac{1}{\ln r} \int_{1}^{r} \frac{d x}{f(x)}=\infty .
$$

Очевидно, что требование

$$
\lim _{r \rightarrow \infty} \frac{f(r)}{r}=0
$$

является достаточным для выполнения (0.39). Для областей вращения, удовлетворяющих условию (0.40), справедливо соотношение (0.33) (см. предложение 2).

Таким образом, для областей вращения, удовлетворяющих условию (0.40), выбор функции $N_{m}(t), m>1$, формулой (0.34) оправдан и справедлива оценка (0.35). Однако для областей вращения можно получить более тонкие оценки.

Пусть $\mathcal{P}(\rho, z)=\left\{\left(x_{1}, x_{2}\right) \in \mathbb{R}_{2} \mid z \leqslant x_{1}<z+\rho, 0<x_{2}<\rho\right\}-$ квадрат со стороной $\rho$ и левой нижней вершиной в точке $z$ оси абсцисс. Для положительной функции $f\left(x_{1}\right), x_{1}>0$, символ $\Gamma_{1}^{r}(f)$ будет обозначать криволинейную трапецию:

$$
\Gamma_{1}^{r}(f)=\left\{\left(x_{1}, x_{2}\right) \in \mathbb{R}_{2} \mid 1 \leqslant x_{1}<r, 0<x_{2}<f\left(x_{1}\right)\right\}
$$

Через $\rho_{*}(r)$ обозначим сторону наибольшего квадрата $\mathcal{P}\left(\rho_{*}, z_{*}\right)$, содержащегося в $\Gamma_{1}^{r}(f)$.

Определим функцию $r_{1}(t), t \geqslant 0$, равенством

$$
\int_{1}^{r_{1}} \frac{d x}{f(x)}=\frac{t}{\rho_{*}^{2}\left(r_{1}\right)}
$$


В силу возрастания функции $\rho_{*}^{2}(r) \int_{1}^{r} \frac{d x}{f(x)}, r \geqslant 1$, равенство (0.41) однозначно определяет монотонно возрастающую функцию $r_{1}(t), t \geqslant 0$.

Следствием теоремы 2 для областей вращения вида (0.36) являются следующие оценки (см. утверждение 5):

$$
\begin{array}{lll}
\text { при } m>1 & \|u(t)\|_{L_{2}(\Omega(f))} \leqslant \widetilde{M}_{m} t^{-1 /(m-1)} g_{m}(t), \quad t \geqslant 1 ; \\
\text { при } m=1 & \|u(t)\|_{L_{2}(\Omega(f))} \leqslant \widetilde{M}_{1} \exp \left(-\widetilde{\varkappa} \int_{1}^{r_{1}(t)} \frac{d x}{f(x)}\right), \quad t \geqslant 0 ;
\end{array}
$$

здесь функция $g_{m}(t)$ растет медленнее любой степенной функции $t^{\gamma}, \gamma>0$.

В области $\Omega\left(f_{a}\right)$ с функцией $f_{a}(x)=x^{a}, 0 \leqslant a<1, x>0$, для решения задачи (0.1)-(0.3) оценки $(0.42),(0.43)$ принимают соответственно следующий вид (см. пример 1):

$$
\begin{gathered}
\|u(t)\|_{L_{2}(\Omega(f))} \leqslant \widetilde{M}_{m, a} t^{-1 /(m-1)}(\ln t)^{\chi /(1-a)}, \\
m>1, \quad t \geqslant e, \quad \chi=a \frac{m+1}{m-1}+a \frac{n-1}{2}+\frac{1}{2} ; \\
\|u(t)\|_{L_{2}(\Omega(f))} \leqslant \widetilde{M}_{1, a} \exp \left(-\widetilde{\varkappa}_{a} t^{(1-a) /(1+a)}\right), \quad m=1, \quad t \geqslant 0 .
\end{gathered}
$$

В области $\Omega(f)$ с функцией $f(x)=e, 0<x<e, f(x)=x / \ln x, x \geqslant e$, для решения задачи (0.1)-(0.3) оценки $(0.42),(0.43)$ принимают соответственно следующий вид (см. пример 2):

$$
\begin{gathered}
\|u(t)\|_{L_{2}(\Omega(f))} \leqslant \widetilde{M}_{m} t^{-1 /(m-1)}(\ln t)^{-\sigma / 2} \exp \left(\varrho(\ln t)^{1 / 2}\right), \\
m>1, \quad t \geqslant e, \quad \sigma=\frac{m+1}{m-1}+\frac{n-1}{2}, \quad \varrho>0 ; \\
\|u(t)\|_{L_{2}(\Omega(f))} \leqslant \widetilde{M}_{1} \exp \left(-\widetilde{\varkappa}(\ln t)^{2}\right), \quad m=1, \quad t \geqslant 1 .
\end{gathered}
$$

\section{$\S 1$. Вспомогательные утверждения}

Пусть $\|\cdot\|_{p, Q}$ - норма в $L_{p}(Q)$, причем значения $p=2, Q=\Omega$ опускаются. Через $D_{a}^{b}=(a, b) \times \Omega$ обозначим цилиндр, значения $a=0$ и $b=\infty$ могут отсутствовать. Банаховы пространства $\stackrel{\circ}{W} \underset{2,1}{1,1}\left(D^{T}\right), \stackrel{\circ}{W_{2}^{0,1}} \underset{2,1}{ }\left(D^{T}\right)$ определим как пополнения пространства $C_{0}^{\infty}\left(D_{-1}^{T+1}\right)$ соответственно по нормам

$$
\begin{gathered}
\|u\|_{W_{2, m+1}^{1,1}\left(D^{T}\right)}=\|u\|_{D^{T}}+\|\nabla u\|_{m+1, D^{T}}+\left\|u_{t}\right\|_{D^{T}}, \\
\|u\|_{W_{2, m+1}^{0,1}\left(D^{T}\right)}=\|u\|_{D^{T}}+\|\nabla u\|_{m+1, D^{T}} .
\end{gathered}
$$

Банахово пространство $\stackrel{\circ}{V} \underset{2, m+1}{0,1}\left(D^{T}\right)$ состоит из всех элементов пространства $\stackrel{\circ}{W} \underset{2, m+1}{0,1}\left(D^{T}\right)$, непрерывных по $t \in[0, T]$ в норме $L_{2}(\Omega)$, с нормой

$$
\|u\|_{V_{2, m+1}^{0,1}\left(D^{T}\right)}=\max _{0 \leqslant t \leqslant T}\|u(t)\|+\|\nabla u\|_{m+1, D^{T}} .
$$


ОПРЕДЕЛЕНИЕ 1. Обобщенным решением задачи (0.1)-(0.3) в $D^{T}$ назовем функцию $u(t, \mathbf{x}) \in \stackrel{\circ}{V} \underset{2,1}{0,1}\left(D^{T}\right)$, удовлетворяющую интегральному тождеству

$$
\int_{D^{T}}\left(-u v_{t}+\sum_{\alpha=1}^{n} a_{\alpha}(t, \mathbf{x}, \nabla u) v_{x_{\alpha}}+a(t, \mathbf{x}, u) v\right) d \mathbf{x} d t=\int_{\Omega} \varphi(\mathbf{x}) v(0, \mathbf{x}) d \mathbf{x}
$$

для любой функции $v(t, \mathbf{x}) \in \stackrel{\circ}{\underset{W}{W}} \underset{2, m+1}{1,1}\left(D^{T}\right)$ такой, что $v(T, \mathbf{x})=0$.

Функция $u(t, \mathbf{x})$ - решение задачи $(0.1)-(0.3)$ в $D$, если при всех $T>0$ она является решением этой задачи в $D^{T}$.

Для произвольного элемента $u(t, \mathbf{x}) \in \stackrel{\circ}{V_{2, m+1}^{0,1}}\left(D^{T}\right)$ справедливо неравенство

$$
\|u\|_{D^{T}, q+1} \leqslant \beta\|u\|_{V_{2, m+1}^{0,1}\left(D^{T}\right)}, \quad q=m+\frac{2(m+1)}{n} .
$$

Неравенство (1.2) выводится так же, как и неравенство (3.4) из [18; гл. II, §3]. Таким образом, пространство $\stackrel{\circ}{V} \underset{2, m+1}{0,1}\left(D^{T}\right)$ вкладывается в $L_{q+1}\left(D^{T}\right)$, а следовательно, и в пространство $L_{q_{*}+1}\left(D^{T}\right), 1 \leqslant q_{*} \leqslant q$. Очевидно, пространство $\stackrel{\circ}{W} \underset{2, m+1}{1,1}\left(D^{T}\right)$ вкладывается в пространство $\stackrel{\circ}{V} \underset{2,1}{0,1}\left(D^{T}\right)$.

Используя условия $(0.5),(0.6),(0.8),(0.9)$ и неравенство Гёльдера, для функции $u(t, \mathbf{x}) \in \stackrel{\circ}{V} \underset{2, m+1}{0,1}\left(D^{T}\right)$ выводим неравенства

$$
\begin{aligned}
& \|\mathbf{a}(t, \mathbf{x}, \nabla u)\|_{(m+1) / m, D^{T}} \leqslant \widehat{a}\left(\int_{D^{T}}|\nabla u|^{m+1} d \mathbf{x} d t\right)^{m /(m+1)}=\widehat{a}\|\nabla u\|_{m+1, D^{T}}^{m}, \\
& \|a(t, \mathbf{x}, u)\|_{\left(q^{*}+1\right) / q *, D^{T}} \leqslant \widetilde{a}\left(\int_{D^{T}}|u|^{q^{*}+1} d \mathbf{x} d t\right)^{q^{*} /\left(q^{*}+1\right)}=\widetilde{a}\|u\|_{q^{*}+1, D^{T}}^{q^{*}}
\end{aligned}
$$

Определение обобщенного решения корректно, поскольку входящие в (1.1) интегралы конечны. Действительно, в силу неравенств Гёльдера и оценок (1.3), (1.4) для функций $u(t, \mathbf{x}), v(t, \mathbf{x}) \in \stackrel{\circ}{V_{2, m+1}^{0,1}}\left(D^{T}\right)$ имеем

$$
\begin{gathered}
\int_{D^{T}} \sum_{\alpha=1}^{n}\left|a_{\alpha}(t, \mathbf{x}, \nabla u)\right|\left|v_{x_{\alpha}}\right| d \mathbf{x} d t \leqslant \int_{D^{T}}|\mathbf{a}(t, \mathbf{x}, \nabla u)||\nabla v| d \mathbf{x} d t \\
\leqslant\|\mathbf{a}\|_{(m+1) / m, D^{T}}\|\nabla v\|_{m+1, D^{T}} \leqslant \widehat{a}\|\nabla u\|_{m+1, D^{T}}^{m}\|\nabla v\|_{m+1, D^{T}}, \\
\int_{D^{T}}|a(t, \mathbf{x}, u)||v| d \mathbf{x} d t \leqslant\|a\|_{\left(q^{*}+1\right) / q^{*}, D^{T}}\|v\|_{q_{*}+1, D^{T}} \leqslant \widetilde{a}\|u\|_{q_{*}+1, D^{T}}^{q_{*}}\|v\|_{q_{*}+1, D^{T}} .
\end{gathered}
$$

УТВЕРЖДЕНИЕ 1. Если выполнены условия (0.4)-(0.9), то обобщенное решение $и(t, \mathbf{x})$ задачи $(0.1)-(0.3)$ существует, единственно и справедливы неравенства

$$
\begin{gathered}
\|u(t)\|^{2}+2 \bar{a} \int_{0}^{t}\|\nabla u(\tau)\|_{m+1}^{m+1} d \tau \leqslant\|\varphi\|^{2}, \quad t \geqslant 0 \\
\frac{d}{d t}\|u(t)\|^{2}+2 \bar{a}\|\nabla u(t)\|_{m+1}^{m+1} \leqslant 0, \quad t>0 .
\end{gathered}
$$


Существование решения задачи (0.1)-(0.3) доказывается методом галёркинских приближений аналогично доказательству соответствующего утверждения в случае ограниченной области (см. [18; гл. 5, теорема 6.7]). При доказательстве единственности решения будет использована

Лемма 1. Если $u(t, \mathbf{x})$ принадлежст пространству $\stackrel{\circ}{V} \underset{2,1}{0,1}\left(D^{T}\right)$, то усреднения

$$
u_{h}(t, \mathbf{x})=\frac{1}{h} \int_{t}^{t+h} u(\tau, \mathbf{x}) d \tau
$$

при $0<h \leqslant \delta<T$ принадлежат пространству $\stackrel{\circ}{W} \underset{2,1}{1,1}\left(D^{T-\delta}\right)$, причем

$$
\left\|u_{h}-u\right\|_{V_{2, m+1}^{0,1}\left(D^{T-\delta}\right)} \rightarrow 0 \quad \text { npu } h \rightarrow 0 .
$$

Если $u(t, \mathbf{x}) \in L_{k}\left(D^{T}\right), k \geqslant 1$, то усреднения $u_{h}$ сходятся $к и$ при $h \rightarrow 0$ в норме $L_{k}\left(D^{T-\delta}\right)$.

Доказательство проводится аналогично доказательству леммы 4.7 из [18; гл. II, § 4].

В тождестве (1.1) положим $v=w_{-h}, 0<h \leqslant \delta<T$, где $w$ принадлежит $\stackrel{\circ}{V} \underset{2,1}{0,1+1}\left(D^{t}\right)$ и продолжена нулем вне интервала $(0, t) \subset(0, T-\delta)$. Выполнив несложные преобразования, установим равенство

$$
\int_{D^{t}}\left\{\left(u_{h}\right)_{\tau} w+(\mathbf{a}(\tau, \mathbf{x}, \nabla u))_{h} \cdot \nabla w+(a(\tau, \mathbf{x}, u))_{h} w\right\} d \mathbf{x} d \tau=0
$$

которое также справедливо для $w \in \stackrel{\circ}{V} \underset{2, m+1}{0,1}\left(D^{t}\right)$. Здесь и далее использовано обозначение $\xi \cdot \eta=\sum_{\alpha=1}^{n} \xi_{\alpha} \eta_{\alpha}, \xi, \eta \in \mathbb{R}_{n}$. Положив $w(\tau, \mathbf{x})=u_{h}(\tau, \mathbf{x})$ и проинтегрировав первое слагаемое, получим

$$
\left.\frac{1}{2}\left\|u_{h}(\tau)\right\|^{2}\right|_{\tau=0} ^{\tau=t}+\int_{D^{t}}\left\{(\mathbf{a}(\tau, \mathbf{x}, \nabla u))_{h} \cdot \nabla u_{h}+(a(\tau, \mathbf{x}, u))_{h} u_{h}\right\} d \mathbf{x} d \tau=0 .
$$

Согласно лемме 1 усреднения Стеклова функций сходятся к самим функциям в $D^{T-\delta}$ в нормах соответствующих пространств. Пользуясь этим, предельным переходом при $h \rightarrow 0$ из последнего равенства для $t \in[0, T-\delta]$ установим тождество

$$
\left.\frac{1}{2}\|u(\tau)\|^{2}\right|_{\tau=0} ^{\tau=t}+\int_{D^{t}}\{\mathbf{a}(\tau, \mathbf{x}, \nabla u) \cdot \nabla u+a(\tau, \mathbf{x}, u) u\} d \mathbf{x} d \tau=0 .
$$

Дифференцируя его по $t$, выводим

$$
\frac{1}{2} \frac{d}{d t}\|u(t)\|^{2}+\int_{\Omega}\{\mathbf{a}(t, \mathbf{x}, \nabla u) \cdot \nabla u+a(t, \mathbf{x}, u) u\} d \mathbf{x}=0 .
$$

Воспользовавшись условиями $(0.4),(0.6),(0.7),(0.9)$ и начальным условием (0.3), из соотношений (1.8), (1.9) выводим неравенства (1.5), (1.6). В силу произвольности $T>0$ можем считать, что неравенства $(1.5),(1.6)$ доказаны при любом $t>0$. 
Докажем единственность обобщенного решения задачи (0.1)-(0.3). Пусть существуют два решения $u^{1}, u^{2}$ задачи (0.1)-(0.3). Введем обозначения $\delta u=u^{1}-u^{2}, \quad \delta \mathbf{a}=\mathbf{a}\left(t, \mathbf{x}, \nabla u^{1}\right)-\mathbf{a}\left(t, \mathbf{x}, \nabla u^{2}\right), \quad \delta a=a\left(t, \mathbf{x}, u^{1}\right)-a\left(t, \mathbf{x}, u^{2}\right)$. Запишем тождество (1.7) дважды для $u^{1}, u^{2}$ и вычтем из первого второе; получим равенство

$$
\int_{D^{t}}\left\{\left(\delta u_{h}\right)_{\tau} w+(\delta \mathbf{a})_{h} \cdot \nabla w+(\delta a)_{h} w\right\} d \mathbf{x} d \tau=0,
$$

в котором положим $w=\delta u_{h}$. После интегрирования первого слагаемого, выполнив предельный переход при $h \rightarrow 0$, для $t \in[0, T-\delta]$ выводим

$$
\left.\frac{1}{2}\|\delta u(\tau)\|^{2}\right|_{\tau=0} ^{\tau=t}+\int_{D^{t}}\{\delta \mathbf{a} \cdot \nabla(\delta u)+\delta a \delta u\} d \mathbf{x} d \tau=0 .
$$

В силу произвольности $T>0$ последнее неравенство справедливо при всех $t \geqslant 0$. Воспользовавшись $(0.4),(0.7)$, из последнего соотношения получаем неравенство

$$
\|\delta u(t)\|^{2}+2 \bar{a} \int_{0}^{t}\|\nabla(\delta u)(\tau)\|_{m+1}^{m+1} d \tau \leqslant 0, \quad t \geqslant 0 .
$$

Следовательно, $\|\delta u(t)\|=0$ при любом $t \geqslant 0$. Утверждение 1 доказано.

\section{§ 2. Оценки сверху}

В этом параграфе будут доказаны предложение 1 и теоремы 1,2 .

Определим монотонно невозрастающую последовательность $\lambda(N)$ :

$$
\lambda(N)=\min \left\{\lambda^{(0)}, \lambda_{1}^{(1)}, \ldots, \lambda_{p^{(1)}}^{(1)}, \lambda_{1}^{(N)}, \ldots, \lambda_{p^{(N)}}^{(N)}\right\}, \quad m \geqslant 1, \quad N=\overline{0, \infty}
$$

Поскольку выполнено условие $(0.21)$, то $\lambda(N)>0, N=\overline{0, \infty}$. Поэтому из определения (0.16) следует неравенство

$$
\lambda(N)\|g\|_{m+1, \Omega^{(N)}}^{m+1} \leqslant\|\nabla g\|_{m+1, \Omega^{(N)}}^{m+1}, \quad g(\mathbf{x}) \in C_{0}^{\infty}(\Omega), \quad m \geqslant 1, \quad N \geqslant 0 .
$$

Применяя неравенство Гёльдера, выводим

$$
\begin{gathered}
\|g\|_{\Omega^{(N)}} \leqslant\|g\|_{m+1, \Omega^{(N)}}\left(\operatorname{mes} \Omega^{(N)}\right)^{(m-1) /(2(m+1))}, \\
g(\mathbf{x}) \in C_{0}^{\infty}(\Omega), \quad m \geqslant 1, \quad N \geqslant 0 .
\end{gathered}
$$

Соединяя последние неравенства, получаем оценку

$$
F_{m}(N) \leqslant \lambda^{-1 /(m+1)}(N)\left(\operatorname{mes} \Omega^{(N)}\right)^{(m-1) /(2(m+1))}, \quad m \geqslant 1, \quad N \geqslant 0 .
$$

ДоКАЗАТЕЛЬСТВО ПРЕДЛОЖЕНИЯ 1. Из (2.2), применяя определения (2.1), (0.17) и условия $(0.29),(0.30)$, выводим

$$
\begin{aligned}
F_{m}(N) & \leqslant C_{1} \max _{i=1, p^{(\nu)}, \nu=1, N}\left\{t_{i}^{(\nu)},\left(\lambda^{(0)}\right)^{-1 /(m+1)}\right\}\left(\operatorname{mes} \Omega^{(N)}\right)^{(m-1) /(2(m+1))} \\
& \leqslant C_{2} a^{N\{1+n(m-1) /(2(m+1))\}}, \quad N \geqslant 1 .
\end{aligned}
$$

Итак, неравенство (0.28) доказано с $b=\ln a\{1+n(m-1) /(2(m+1))\}$. 
ДоКАЗАТЕЛЬСтво теоремЫ 1 . Зафиксируем натуральное число $N \geqslant 2$. Выберем число $\varkappa>0$ так, чтобы $\theta^{1 /(m+1)} \varkappa e^{\varkappa} \widehat{a} \leqslant \bar{a}$. Построим определенную в $\Omega$ липшицеву функцию $\xi(\mathbf{x})$, удовлетворяющую условиям

$$
\xi(\mathbf{x})= \begin{cases}0, & \mathbf{x} \in \overline{\Omega^{(0)}}, \\ \exp (-\varkappa(N-1)) \min \left(1, \frac{\operatorname{dist}\left(\widetilde{S}_{i}^{(0)}, \mathbf{x}\right)}{t_{i}^{(1)}}\right), & \mathbf{x} \in \omega_{i}^{(1)}, i=\overline{1, p^{(1)},} \\ \exp (-\varkappa(N+1-\nu)) \exp \left(\varkappa \min \left(1, \frac{\operatorname{dist}\left(\widetilde{S}_{i}^{(\nu-1)}, \mathbf{x}\right)}{t_{i}^{(\nu)}}\right)\right), & \mathbf{x} \in \omega_{i}^{(\nu)}, i=\overline{1, p^{(\nu)}}, \nu=\overline{2, N}, \\ 1, & \mathbf{x} \in \Omega \backslash \Omega^{(N)} .\end{cases}
$$

Нетрудно установить следующие соотношения:

$$
\begin{aligned}
& |\nabla \xi| \leqslant \frac{\exp (-\varkappa(N-1))}{t_{i}^{(1)}}, \quad \mathbf{x} \in \omega_{i}^{(1)}, \quad i=\overline{1, p^{(1)}} \\
& |\nabla \xi| \leqslant \frac{\varkappa \xi}{t_{i}^{(\nu)}}, \quad \mathbf{x} \in \omega_{i}^{(\nu)}, \quad i=\overline{1, p^{(\nu)}}, \quad \nu=\overline{2, N} \\
& \max _{\omega_{i}^{(\nu)}} \xi(\mathbf{x})=e^{\varkappa} \min _{\omega_{i}^{(\nu)}} \xi(\mathbf{x}), \quad i=\overline{1, p^{(\nu)}}, \quad \nu=\overline{2, N} \\
& \max _{\Omega_{(0)}^{(1)}} \xi(\mathbf{x})=\exp (-\varkappa(N-1)) \text {. }
\end{aligned}
$$

Положим в (1.7)

$$
w(\tau, \mathbf{x})=u_{h}(\tau, \mathbf{x}) \xi(\mathbf{x}) \in \stackrel{\circ}{W}_{2, m+1}^{1,1}\left(D^{t}\right), \quad 0<h \leqslant \delta, \quad 0<t \leqslant T-\delta .
$$

Интегрирование первого слагаемого приводит к равенству

$$
\left.\frac{1}{2} \int_{\Omega} u_{h}^{2} \xi\right|_{\tau=0} ^{\tau=t} d \mathbf{x}+\int_{D^{t}}\left\{(\mathbf{a}(\tau, \mathbf{x}, \nabla u))_{h} \cdot \nabla\left(u_{h} \xi\right)+(a(\tau, \mathbf{x}, u))_{h} u_{h} \xi\right\} d \mathbf{x} d \tau=0
$$

Перейдя (на основе леммы 1) в последнем равенстве к пределу при $h \rightarrow 0$, получим

$$
\left.\frac{1}{2} \int_{\Omega} u^{2} \xi\right|_{\tau=0} ^{\tau=t} d \mathbf{x}+\int_{D^{t}}\{\mathbf{a}(\tau, \mathbf{x}, \nabla u) \cdot \nabla(u \xi)+a(\tau, \mathbf{x}, u) u \xi\} d \mathbf{x} d \tau=0
$$

Далее, используя условия (0.4)-(0.7), (0.9), получаем (с учетом того, что $\xi \varphi=0)$

$$
\frac{1}{2} \int_{\Omega} u^{2}(t, \mathbf{x}) \xi(\mathbf{x}) d \mathbf{x}+\bar{a} \int_{D^{t}} \xi|\nabla u|^{m+1} d \mathbf{x} d \tau \leqslant \widehat{a} \int_{D^{t}}|u||\nabla u|^{m}|\nabla \xi| d \mathbf{x} d \tau \equiv I^{t}
$$


Используя (2.3), (2.4), оценим интеграл $I^{t}$ :

$$
\begin{aligned}
I^{t}= & \widehat{a} \int_{0}^{t} \int_{\Omega_{(0)}^{(1)}}|u||\nabla u|^{m}|\nabla \xi| d \mathbf{x} d \tau+\widehat{a} \sum_{\nu=2}^{N} \int_{0}^{t} \int_{\Omega_{(\nu-1)}^{(\nu)}}|u||\nabla u|^{m}|\nabla \xi| d \mathbf{x} d \tau \\
\leqslant & \widehat{a} \sum_{i=1}^{p^{(1)}} \int_{0}^{t} \int_{\omega_{i}^{(1)}}|u||\nabla u|^{m} \frac{\exp (-\varkappa(N-1))}{t_{i}^{(1)}} d \mathbf{x} d \tau \\
& +\widehat{a} \sum_{\nu=2}^{N} \sum_{i=1}^{p^{(\nu)}} \int_{0}^{t} \int_{\omega_{i}^{(\nu)}}|u||\nabla u|^{m} \frac{\varkappa \xi}{t_{i}^{(\nu)}} d \mathbf{x} d \tau .
\end{aligned}
$$

Установим соотношения

$$
\int_{\omega_{i}^{(\nu)}} \frac{|u||\nabla u|^{m}}{t_{i}^{(\nu)}} d \mathbf{x} \leqslant \theta^{1 /(m+1)} \int_{\omega_{i}^{(\nu)}}|\nabla u|^{m+1} d \mathbf{x}, \quad i=\overline{1, p^{(\nu)}}, \quad \nu=\overline{1, \infty} .
$$

Используя неравенство Юнга и применив определение $\lambda$-разбиения $(0.16),(0.17)$, для $i=\overline{1, p^{(\nu)}}, \nu=\overline{1, \infty}$ выводим неравенства

$$
\begin{aligned}
\int_{\omega_{i}^{(\nu)}} & \frac{|u||\nabla u|^{m}}{t_{i}^{(\nu)}} d \mathbf{x} \leqslant \int_{\omega_{i}^{(\nu)}} \frac{m \varepsilon^{1 / m}|\nabla u|^{m+1}}{m+1} d \mathbf{x}+\int_{\omega_{i}^{(\nu)}} \frac{|u|^{m+1}}{(m+1)\left(t_{i}^{(\nu)}\right)^{m+1} \varepsilon} d \mathbf{x} \\
& \leqslant \int_{\omega_{i}^{(\nu)}} \frac{m \varepsilon^{1 / m}|\nabla u|^{m+1}}{m+1} d \mathbf{x}+\int_{\omega_{i}^{(\nu)}} \frac{\theta|u|^{m+1} \lambda_{i}^{(\nu)}}{(m+1) \varepsilon} d \mathbf{x} \\
& \leqslant \frac{1}{m+1} \int_{\omega_{i}^{(\nu)}}\left(m \varepsilon^{1 / m}+\frac{\theta}{\varepsilon}\right)|\nabla u|^{m+1} d \mathbf{x} .
\end{aligned}
$$

Выбрав $\varepsilon=\theta^{m /(m+1)}$, получаем (2.9). В силу (2.5) из (2.9) получаем неравенства

$$
\int_{\omega_{i}^{(\nu)}} \frac{\xi|u||\nabla u|^{m}}{t_{i}^{(\nu)}} d \mathbf{x} \leqslant e^{\varkappa} \theta^{1 /(m+1)} \int_{\omega_{i}^{(\nu)}} \xi|\nabla u|^{m+1} d \mathbf{x}, \quad i=\overline{1, p^{(\nu)}}, \quad \nu=\overline{2, N} .
$$

Используя (2.8), (2.9) и последнее соотношение, нетрудно привести (2.7) к виду

$$
\begin{aligned}
& \frac{1}{2} \int_{\Omega} \xi u^{2}(t, \mathbf{x}) d \mathbf{x}+\bar{a} \int_{0}^{t} \int_{\Omega} \xi|\nabla u|^{m+1} d \mathbf{x} d \tau \\
& \leqslant \widehat{a} \theta^{1 /(m+1)} \exp (-\varkappa(N-1)) \int_{0}^{t} \int_{\Omega_{(0)}^{(1)}}|\nabla u|^{m+1} d \mathbf{x} d \tau \\
& \quad+\widehat{a} \varkappa \theta^{1 /(m+1)} e^{\varkappa} \sum_{\nu=2}^{N} \int_{0}^{t} \int_{\Omega_{(\nu-1)}^{(\nu)}} \xi|\nabla u|^{m+1} d \mathbf{x} d \tau
\end{aligned}
$$

В силу выбора числа $\varkappa$ устанавливаем, что

$$
\int_{\Omega \backslash \Omega^{(N)}} u^{2}(t, \mathbf{x}) d \mathbf{x} \leqslant 2 \frac{\bar{a}}{\varkappa} \exp (-\varkappa N) \int_{0}^{t} \int_{\Omega_{(0)}^{(1)}}|\nabla u|^{m+1} d \mathbf{x} d \tau .
$$


Соединяя последнюю оценку с $(1.5)$ и полагая $\varkappa=2 \varkappa_{m}$, выводим неравенство (0.22). При $N=0,1$ справедливости оценки (0.22) нетрудно добиться подбором константы $M$ благодаря ограниченности сверху левой и снизу правой частей.

ДОКАЗАТЕЛЬСТВо ТЕОРЕмЫ 2. Выберем произвольное целое неотрицательное число $N \geqslant 0$. Согласно теореме 1 , вводя обозначение $\varepsilon(N)=M^{2} e^{-2 \varkappa_{m} N}\|\varphi\|^{2}$, получаем соотношение

$$
\|u(t)\|^{2} \leqslant\|u(t)\|_{\Omega^{(N)}}^{2}+\varepsilon(N), \quad t \geqslant 0 .
$$

Из определения (0.23) следует, что

$$
\|u(t)\|^{2} \leqslant F_{m}^{2}(N)\|\nabla u(t)\|_{m+1, \Omega^{(N)}}^{2}+\varepsilon(N), \quad t \geqslant 0 .
$$

Обозначим через $t_{N}$ точку интервала $[0, \infty)$ такую, что $E(t)=\|u(t)\|^{2}=\varepsilon(N)$. В случае если $E(t)>\varepsilon(N)$ при любом $t \geqslant 0$, считаем, что $t_{N}=\infty$. Очевидно, для $t \in\left[0, t_{N}\right)$ справедливо неравенство $E(t)>\varepsilon(N)$; тогда соотношение $(2.10)$ можно переписать в виде

$$
(E(t)-\varepsilon(N))^{(m+1) / 2} \leqslant F_{m}^{m+1}(N)\|\nabla u(t)\|_{m+1, \Omega^{(N)}}^{m+1}, \quad t \in\left[0, t_{N}\right) .
$$

Соединяя (2.11) с (1.6), выводим соотношение

$$
F_{m}^{m+1}(N) \frac{d E(t)}{d t} \leqslant-2 \bar{a}(E(t)-\varepsilon(N))^{(m+1) / 2}, \quad t \in\left(0, t_{N}\right) .
$$

Решая дифференциальное неравенство при $m>1$, получаем

$$
(E(t)-\varepsilon(N))^{(m-1) / 2} \leqslant C_{1} \frac{F_{m}^{m+1}(N)}{t}, \quad t \in\left(0, t_{N}\right) .
$$

Подставив в последнее неравенство значение $\varepsilon(N)$, для $t \in\left(0, t_{N}\right)$ выводим

$$
E(t) \leqslant C_{2} F_{m}^{2(m+1) /(m-1)}(N) t^{-2 /(m-1)}+M^{2} \exp \left(-2 \varkappa_{m} N\right)\|\varphi\|^{2} .
$$

Отметим, что для $t \in\left[t_{N}, \infty\right)$ выполнено неравенство $E(t) \leqslant \varepsilon(N)$, и оценка (2.13) также справедлива.

В (2.13) положим $N=N_{m}(t)$ и воспользуемся определением (0.24) функции $N_{m}(t)$, в итоге получаем

$$
\begin{aligned}
E(t) & \leqslant C_{2} t^{-2 /(m-1)} F_{m}^{2(m+1) /(m-1)}\left(N_{m}(t)\right)+M^{2} \exp \left(-2 \varkappa_{m} N_{m}(t)\right)\|\varphi\|^{2} \\
& \leqslant C_{3} t^{-2 /(m-1)} F_{m}^{2(m+1) /(m-1)}\left(N_{m}(t)\right), \quad t>0 .
\end{aligned}
$$

Таким образом, для $m>1$ установлено неравенство (0.26).

При $m=1$ неравенство (2.12) принимает вид

$$
\frac{d E(t)}{d t} \leqslant-\frac{2 \bar{a}}{F_{1}^{2}(N)}(E(t)-\varepsilon(N)), \quad t \in\left(0, t_{N}\right) .
$$

Решая это дифференциальное неравенство, будем иметь

$$
E(t) \leqslant M^{2} \exp \left(-2 \varkappa_{1} N\right)\|\varphi\|^{2}+\exp \left(-2 \bar{a} \frac{t}{F_{1}^{2}(N)}\right)\|\varphi\|^{2}, \quad t>0 .
$$


Полагая $N=N_{1}(t)$ и применяя определение $(0.25)$, для $t \geqslant 0$ получаем

$$
E(t) \leqslant\left(M^{2}+1\right) \exp \left(-2 \varkappa^{*} N_{1}(t)\right)\|\varphi\|^{2}, \quad \varkappa^{*}=\min \left(\varkappa_{1}, \bar{a}\right) .
$$

Тем самым, установлено неравенство (0.27).

\section{§ 3. $\lambda$-последовательности}

В этом параграфе доказывается необходимое и достаточное условие существования $\lambda$-последовательности.

Лемма 2. Пусть $z, a, b, c-$ такие действительные числа, что $0<z \leqslant$ $a<b \leqslant c, \Delta=(z, a) \cup(b, c),|\Delta|=a-z+c-b$. Тогда для любой функции $g(x) \in C_{0}^{\infty}(\mathbb{R})$ при $k \geqslant 1$ справедливо неравенство

$$
\|g\|_{k, \Delta} \leqslant\left(\frac{c-z}{b-a}\right)^{1 / k}\|g\|_{k,(a, b)}+(c-z)\left\|g^{\prime}\right\|_{k,(z, c)} .
$$

ДокАЗАТЕЛЬСтво. Из формулы Ньютона-Лейбница следует неравенство

$$
|g(x)| \leqslant|g(y)|+\int_{y}^{x}\left|g^{\prime}(t)\right| d t, \quad a \leqslant y<x \leqslant c .
$$

Используя неравенство Гёльдера, оценим интеграл:

$$
\int_{y}^{x} 1 \cdot\left|g^{\prime}(t)\right| d t \leqslant(x-y)^{(k-1) / k}\left\|g^{\prime}\right\|_{k,(y, x)} .
$$

Тогда неравенство (3.2) можем записать в виде

$$
\begin{gathered}
|g(x)| \leqslant|g(y)|+(x-y)^{(k-1) / k}\left\|g^{\prime}\right\|_{k,(y, x)} \leqslant|g(y)|+(c-a)^{(k-1) / k}\left\|g^{\prime}\right\|_{k,(a, c)}, \\
a \leqslant y<x \leqslant c .
\end{gathered}
$$

Применив интегральное неравенство Минковского для $x \in(b, c)$ и $y \in(a, b)$, будем иметь

$$
(b-a)^{1 / k}\|g\|_{k,(b, c)} \leqslant(c-b)^{1 / k}\left\{\|g\|_{k,(a, b)}+(c-a)^{(k-1) / k}(b-a)^{1 / k}\left\|g^{\prime}\right\|_{k,(a, c)}\right\} .
$$

Аналогично устанавливается неравенство

$$
(b-a)^{1 / k}\|g\|_{k,(z, a)} \leqslant(a-z)^{1 / k}\left\{\|g\|_{k,(a, b)}+(b-z)^{(k-1) / k}(b-a)^{1 / k}\left\|g^{\prime}\right\|_{k,(z, b)}\right\} .
$$

Объединив (3.4) и (3.5), можем записать

$$
(b-a)^{1 / k}\|g\|_{k, \Delta} \leqslant|\Delta|^{1 / k}\left\{\|g\|_{k,(a, b)}+(c-z)^{(k-1) / k}(b-a)^{1 / k}\left\|g^{\prime}\right\|_{k,(z, c)}\right\} .
$$

Учитывая, что $|\Delta|<c-z$, из последнего выводим (3.1).

УтВеРЖДЕНИЕ 2. Пусть $0<z \leqslant a<b \leqslant c, k=m+1, m \geqslant 1$. Тогда

$$
\lambda^{-1 / k}(z, c) \leqslant\left(\frac{c-z}{b-a}+1\right)^{1 / k} \lambda^{-1 / k}(a, b)+(c-z) .
$$


Если дополнительно выполнено условие

$$
2 \leqslant \lambda(a, b)(b-a)(c-z)^{m}
$$

mo

$$
1 \leqslant 2^{k}(c-z)^{k} \lambda(z, c)
$$

ДокАЗАтЕльство. Применим неравенство (3.1) к интервалам $(a, b) \subset(z, c)$ и функции $g\left(x_{1}, \mathbf{x}^{\prime}\right) \in C_{0}^{\infty}(\Omega)$, продолженной нулем вне $\Omega$, по переменной $x_{1}$ в следующем виде:

$$
\left\|g\left(\mathbf{x}^{\prime}\right)\right\|_{k, \Delta} \leqslant\left(\frac{c-z}{b-a}\right)^{1 / k}\left\|g\left(\mathbf{x}^{\prime}\right)\right\|_{k,(a, b)}+(c-z)\left\|D_{x_{1}} g\left(\mathbf{x}^{\prime}\right)\right\|_{k,(z, c)}, \quad \mathbf{x}^{\prime} \in \mathbb{R}_{n-1} .
$$

Применяя интегральное неравенство Минковского по $\mathbf{x}^{\prime} \in \mathbb{R}_{n-1}$, находим, что

$$
\|g\|_{k, \Omega_{z}^{a} \cup \Omega_{b}^{c}} \leqslant\left(\frac{c-z}{b-a}\right)^{1 / k}\|g\|_{k, \Omega_{a}^{b}}+(c-z)\|\nabla g\|_{k, \Omega_{z}^{c}} .
$$

Используя неравенство Минковского для сумм, выводим

$$
\|g\|_{k, \Omega_{z}^{c}} \leqslant\left(\frac{c-z}{b-a}+1\right)^{1 / k}\|g\|_{k, \Omega_{a}^{b}}+(c-z)\|\nabla g\|_{k, \Omega_{z}^{c}}
$$

Применив определение (0.16), получим неравенство (3.6).

Учитывая, что $c-z>b-a$, из (3.6) получаем

$$
\lambda^{-1 / k}(z, c) \leqslant\left[\left(\frac{2}{\lambda(a, b)(b-a)(c-z)^{k-1}}\right)^{1 / k}+1\right](c-z) .
$$

Из последнего неравенства и условия (3.7) следует (3.8).

СлЕДСТвИЕ. Если область удовлетворяет условию (0.19), то $\lambda$-последовательность $\left\{z_{N}\right\}_{N=0}^{\infty}$ существует при произвольном $z_{0}>0$ с числом $\theta \geqslant 2^{m+1}$.

ДокАЗАТЕЛЬство. Пусть $z_{N-1}$ - элемент $\lambda$-последовательности с числом $\theta \geqslant 2^{m+1}$ и по предположению (0.19) $\lambda\left(z_{N-1}, z_{*}\right)>0$. В качестве следующего элемента $\lambda$-последовательности можно взять произвольное $z_{N} \geqslant z_{*}$, удовлетворяющее неравенству $2 \leqslant \lambda\left(z_{N-1}, z_{*}\right)\left(z_{*}-z_{N-1}\right)\left(z_{N}-z_{N-1}\right)^{m}$. Тогда соотношение (0.18) следует из утверждения 2 при $a=z=z_{N-1}, b=z_{*}, c=z_{N}$.

\section{§4. Оценки сверху для областей вращения}

Лемма 3. Рассмотрим область $\Pi_{a}^{b+}=\left\{(x, y) \in \mathbb{R}_{2} \mid a<x<b, y>0\right\} u$ функцию $g(x, y) \in C_{0}^{\infty}\left(\mathbb{R}_{2}\right)$, равную нулю в окрестности луча $\left\{(x, y) \in \mathbb{R}_{2} \mid\right.$ $x=a, y>h\}$. При $k \geqslant 1$ справедливо обобщенное неравенство ФридрихсаСтеклова

$$
\|g\|_{k, \Pi_{a}^{b+}} \leqslant 3^{1 / k}(b-a)\left\|D_{x} g\right\|_{k, \Pi_{a}^{b+}}+2 h\left\|D_{y} g\right\|_{k, \Pi_{a}^{b+}} .
$$


ДокАЗАТЕЛЬСтво. В силу того, что $g(a, y)=0$ при $y>h$, неравенство $(3.3)$ запишем в виде

$$
|g(x, y)| \leqslant(b-a)^{(k-1) / k}\left\|D_{x} g(y)\right\|_{k,(a, b)}, \quad a \leqslant x \leqslant b .
$$

Из последнего неравенства выводим

$$
\|g\|_{k,(a, b) \times(h, \infty)} \leqslant(b-a)\left\|D_{x} g\right\|_{k,(a, b) \times(h, \infty)} .
$$

Далее, при каждом $x \in(a, b)$ из неравенства (3.1) при $z=0, a=h, b=c=2 h$ получаем

$$
\|g(x)\|_{k,(0, h)} \leqslant 2^{1 / k}\|g(x)\|_{k,(h, 2 h)}+2 h\left\|D_{y} g(x)\right\|_{k,(0,2 h)} .
$$

Пользуясь интегральным неравенством Минковского по $x \in(a, b)$, выводим

$$
\|g\|_{k,(a, b) \times(0, h)} \leqslant 2^{1 / k}\|g\|_{k,(a, b) \times(h, 2 h)}+2 h\left\|D_{y} g\right\|_{k,(a, b) \times(0,2 h)} .
$$

Объединяя неравенства (4.2) и (4.3) и пользуясь неравенством Минковского для сумм, установим неравенство (4.1).

УтвеРЖДЕНИЕ 3. П-последовательность $\left\{z_{j}\right\}_{j=0}^{\infty}$ функции $f(x)$ является $\lambda$-последовательностью для области $\Omega(f)$.

ДокАЗАТЕЛЬСтво. Для каждого $s=\overline{2, n}$ рассмотрим область типа слоя

$$
\Omega[f, s]=\left\{\left(x_{1}, \mathbf{x}^{\prime}\right) \in \mathbb{R}_{n}\left|x_{1}>0,\right| x_{s} \mid<f\left(x_{1}\right)\right\}
$$

с положительной функцией $f\left(x_{1}\right)$.

Зафиксируем номер $j \in \mathbb{N}$. Функцию $g\left(x_{1}, \mathbf{x}^{\prime}\right) \in C_{0}^{\infty}(\Omega[f, s])$ продолжим на все $\mathbb{R}_{n}$ нулем за пределы $\Omega[f, s]$. Пусть точка $\widehat{z}_{j} \in\left[z_{j-1}, z_{j}\right]$ такая, что $\inf _{\left[z_{j-1}, z_{j}\right]} f(x)=f\left(\widehat{z}_{j}\right)$; тогда из $(0.37)$ следует

$$
f\left(\widehat{z}_{j}\right) \leqslant \Delta_{j}
$$

Введем обозначения

$$
\begin{gathered}
\Pi_{a}^{b}=\left\{\left(x_{1}, x_{s}\right) \in \mathbb{R}_{2} \mid a<x_{1}<b\right\}, \\
\Pi_{a}^{b \pm}=\left\{\left(x_{1}, x_{s}\right) \in \mathbb{R}_{2} \mid a<x_{1}<b, \pm x_{s}>0\right\} .
\end{gathered}
$$

Воспользовавшись неравенством (4.1) при $k=m+1, m \geqslant 1$ для полуполосы $\Pi_{z_{j-1}}^{\widehat{z}_{j}+}$, получим

$$
\left\|g\left(\mathbf{x}^{\prime \prime}\right)\right\|_{k, \Pi_{z_{j-1}}^{\hat{z}_{j}+}} \leqslant 2 \Delta_{j}\left\|D_{x_{1}} g\left(\mathbf{x}^{\prime \prime}\right)\right\|_{k, \Pi_{z_{j-1}}^{\hat{z}_{j}+}}+2 f\left(\widehat{z}_{j}\right)\left\|D_{x_{s}} g\left(\mathbf{x}^{\prime \prime}\right)\right\|_{k, \Pi_{z_{j-1}}^{\hat{z}_{j}+}}
$$

$x^{\prime \prime}=\left(x_{2}, \ldots, x_{s-1}, x_{s+1}, \ldots, x_{n}\right) \in \mathbb{R}_{n-2}$. Применив $(4.5)$, выводим

$$
\left\|g\left(\mathbf{x}^{\prime \prime}\right)\right\|_{k, \Pi_{z_{j-1}}^{\hat{z}_{j}+}}^{k} \leqslant 4^{k} \Delta_{j}^{k}\left\|\nabla g\left(\mathbf{x}^{\prime \prime}\right)\right\|_{k, \Pi_{z_{j-1}}^{\hat{z}_{j}+}} .
$$


Установив аналогичные неравенства для областей $\Pi_{z_{j-1}}^{\widehat{z}_{j}-}, \Pi_{\widehat{z}_{j}}^{z_{j}+}, \Pi_{\widehat{z}_{j}}^{z_{j}-}$, сложив эти четыре неравенства и проинтегрировав по $x^{\prime \prime}$, получим соотношение

$$
\|g\|_{k, \Omega_{z_{j-1}}^{z_{j}}[f, s]}^{k} \leqslant 4^{k} \Delta_{j}^{k}\|\nabla g\|_{k, \Omega_{z_{j-1}}^{z_{j}}[f, s]^{\circ}}^{k}
$$

Из него следует оценка

$$
1 \leqslant 4^{m+1} \Delta_{j}^{m+1} \lambda\left(z_{j-1}, z_{j} ; \Omega[f, s]\right) .
$$

Поскольку $\Omega(f) \subset \Omega[f, s]$, то $\lambda(a, b ; \Omega[f, s]) \leqslant \lambda(a, b ; \Omega(f))$. Это следует из того, что для $\Omega(f)$ сужается множество, по которому берется инфимум в $(0.16)$. Утверждение доказано.

УТВЕРЖДЕНИЕ 4. Пустъ выполнены условия (0.4)-(0.9), (0.20). Тогда найдутся положительные числа $\varkappa_{m}, \widetilde{M}$ такие, что обобщенное решение $u(t, \mathbf{x})$ задачи (0.1)-(0.3) в цилиндрической области $D(f)=(0, \infty) \times \Omega(f)$ при всех $t \geqslant 0, r \geqslant 1$ удовлетворяет оценке $(0.38)$.

ДокАЗАТЕЛЬСтво. Пусть $\left\{z_{j}\right\}_{j=0}^{\infty}-$ П-последовательность функции $f$. Согласно определению (0.37) справедливы неравенства

$$
\inf _{\left[z_{j-1}, z_{j}\right)} f(x) \geqslant \Delta_{j}, \quad j=\overline{1, \infty},
$$

из которых следуют соотношения

$$
\int_{z_{j-1}}^{z_{j}} \frac{d x}{f(x)} \leqslant \frac{z_{j}-z_{j-1}}{\inf _{\left[z_{j-1}, z_{j}\right)} f(x)} \leqslant 1, \quad j=\overline{1, \infty} .
$$

Суммируя их по $j=\overline{1, N+1}$, выводим

$$
\int_{1}^{z_{N+1}} \frac{d x}{f(x)} \leqslant N+1, \quad N \geqslant 0 .
$$

Пусть $r \in\left[z_{N}, z_{N+1}\right)$; согласно (4.7) установим

$$
\int_{1}^{r} \frac{d x}{f(x)} \leqslant \int_{1}^{z_{N+1}} \frac{d x}{f(x)} \leqslant N+1 .
$$

Соединяя (4.8) и (0.22), выводим оценку (0.38).

При $m>1$ определим монотонно возрастающие функции:

$$
\begin{gathered}
G_{m}(r)=\left(\operatorname{mes} \Omega^{r}\right)^{(m-1) /(2(m+1))} \rho_{*}(r), \quad r \geqslant 1 ; \\
r_{m}(t): \quad \int_{1}^{r_{m}} \frac{d x}{f(x)}=\frac{\ln t}{\varkappa_{m}(m-1)}, \quad t \geqslant 1 .
\end{gathered}
$$

УТВЕРЖДЕНИЕ 5. Пустъ выполнены условия (0.4)-(0.9), (0.20). Тогда существуют положительные числа $\widetilde{M}_{m}, \widetilde{\varkappa}$ такие, что для решения $u(t, \mathbf{x})$ задачи (0.1)-(0.3) в иилиндрической области $D(f)=(0, \infty) \times \Omega(f)$ с функцией $f(x)$, удовлетворяющей условию (0.39), справедливы оченки $(0.42),(0.43)$. 
ДоказАтельство. Положим $\delta(N)=\max _{j=\overline{1, N}} \Delta_{j}, N \in \mathbb{N}$. В силу того, что П-последовательность функции $f$ является $\lambda$-последовательностью для области $\Omega(f)$, согласно (0.18) имеем

$$
\rho_{*}^{m+1}\left(z_{N}\right) \geqslant \delta^{m+1}(N)=\max _{j=1, N} \Delta_{j}^{m+1} \geqslant \frac{1}{\theta \min _{j=1, N} \lambda\left(z_{j-1}, z_{j}\right)} .
$$

Поскольку $\lambda\left(0, z_{0}\right)>0$, то можно подобрать $\epsilon, 0<\epsilon<1$, такое, что

$$
\rho_{*}^{m+1}\left(z_{N}\right) \geqslant \delta^{m+1}(N) \geqslant \delta^{m+1}(1) \geqslant \frac{\epsilon}{\theta \lambda\left(0, z_{0}\right)} .
$$

В итоге будет установлено неравенство

$$
\lambda(N)=\min _{j=\overline{1, N}}\left\{\lambda\left(0, z_{0}\right), \lambda\left(z_{j-1}, z_{j}\right)\right\} \geqslant \frac{\epsilon}{\theta \delta^{m+1}(N)} \geqslant \frac{\beta}{\rho_{*}^{m+1}\left(z_{N}\right)}, \quad N \geqslant 1 .
$$

Далее, из (2.2), (4.11) следует оценка

$$
F_{m}(N) \leqslant \gamma \rho_{*}\left(z_{N}\right)\left(\operatorname{mes} \Omega^{z_{N}}\right)^{(m-1) /(2(m+1))}=\gamma G_{m}\left(z_{N}\right), \quad N \geqslant 1 .
$$

Пусть $r \in\left[z_{N}, z_{N+1}\right)$; тогда из (4.12) получаем

$$
F_{m}(N) \leqslant \gamma\left(\operatorname{mes} \Omega^{r}\right)^{(m-1) /(2(m+1))} \rho_{*}(r)=\gamma G_{m}(r) .
$$

Зафиксируем

$$
t \geqslant \exp \left(\varkappa_{m}(m-1) \int_{1}^{z_{1}} \frac{d x}{f(x)}\right)
$$

и положим $r=r_{m}(t), m>1$. Подставляя (4.13), (4.8) в (2.13) и используя определение (4.10) функции $r_{m}(t)$, получаем при $m>1$ соотношения

$$
\begin{aligned}
\|u(t)\|_{\Omega(f)}^{2} & \leqslant C_{4} \exp \left(-2 \varkappa_{m} \int_{1}^{r_{m}(t)} \frac{d x}{f(x)}\right)+C_{4} G_{m}^{2(m+1) /(m-1)}\left(r_{m}(t)\right) t^{-2 /(m-1)} \\
& \leqslant C_{5} G_{m}^{2(m+1) /(m-1)}\left(r_{m}(t)\right) t^{-2 /(m-1)},
\end{aligned}
$$

откуда следует неравенство $(0.42)$ с функцией $g_{m}(t)=G_{m}^{(m+1) /(m-1)}\left(r_{m}(t)\right)$.

Покажем, что функция $g_{m}(t)$ растет медленнее степенной функции. Из условия $(0.39)$ следует, что для любого $\varepsilon \in(0,1)$ существует $r_{0}$ такое, что

$$
\varepsilon \varkappa_{m}(m-1) \int_{1}^{r} \frac{d x}{f(x)}>\ln r, \quad r \geqslant r_{0} .
$$

Тогда из определения (4.10) функции $r_{m}(t)$ следует, что справедливо неравенство

$$
r_{m}(t)<t^{\varepsilon}, \quad t \geqslant t_{0} .
$$

Далее, из определения (4.9), применяя формулу

$$
\operatorname{mes} \Omega^{r}(f)=c_{n} \int_{0}^{r} f^{n-1}(x) d x,
$$


получаем неравенства

$$
\begin{aligned}
G_{m}(r) & \leqslant C_{6} \rho_{*}(r)\left(\int_{0}^{r} f^{n-1}(x) d x\right)^{(m-1) /(2(m+1))} \\
& \leqslant C_{6} r^{1+(m-1) /(2(m+1))} \max _{[0, r]} f^{(n-1)(m-1) /(2(m+1))}(x), \quad r \geqslant 1 .
\end{aligned}
$$

Применяя следствие неравенства (0.39)

$$
f(x) \leqslant C x, \quad x \geqslant r_{0},
$$

выводим

$$
G_{m}(r) \leqslant C_{7}\left(r_{0}\right) r^{1+n(m-1) /(2(m+1))}, \quad r \geqslant r_{0} .
$$

Соединяя (4.14) и (4.16), устанавливаем, что функция $g_{m}(t)$ растет медленнее любой степени $t$.

Зафиксируем

$$
t \geqslant \rho_{*}^{2}\left(z_{1}\right) \exp \left(\int_{1}^{z_{1}} \frac{d x}{f(x)}\right)
$$

и положим $r=r_{1}(t)$. Подставив (4.13), (4.8) в (2.14) и используя определение (0.41) функции $r_{1}(t)$, выводим

$$
\begin{aligned}
\|u(t)\|_{\Omega(f)}^{2} & \leqslant\left[C_{8} \exp \left(-2 \varkappa_{1} \int_{1}^{r_{1}(t)} \frac{d x}{f(x)}\right)+\exp \left(-\frac{2 \bar{a} t}{\gamma^{2} \rho_{*}^{2}(r(t))}\right)\right]\|\varphi\|_{\Omega(f)}^{2} \\
& \leqslant C_{9} \exp \left(-2 \widetilde{\varkappa} \int_{1}^{r_{1}(t)} \frac{d x}{f(x)}\right)\|\varphi\|_{\Omega(f)}^{2},
\end{aligned}
$$

откуда следует оценка (0.43).

ПРЕДлОЖЕНИЕ 2. Для областей вращения с функиией $f$, удовлетворяющей условию (0.40), справедливо соотношение (0.33).

ДокАЗАТЕЛЬСтво. Пользуясь неравенствами (4.12) и (4.16), выводим

$$
F_{m}(N) \leqslant C_{10}\left(N_{0}\right) z_{N}^{1+n(m-1) /(2(m+1))}, \quad N \geqslant N_{0} .
$$

В силу условия $(0.40)$ можно зафиксировать любое число $\varepsilon \in(0,1)$ такое, что

$$
f(x) \leqslant \varepsilon x, \quad x \geqslant z_{N}, \quad N \geqslant N_{0} .
$$

Тогда, применяя (4.6), получаем

$$
\frac{z_{N+1}}{z_{N}}=\frac{z_{N}+\Delta_{N+1}}{z_{N}} \leqslant 1+\varepsilon, \quad N \geqslant N_{0},
$$

следовательно, справедлива оценка

$$
z_{N} \leqslant(1+\varepsilon)^{N-N_{0}} z_{N_{0}} \leqslant \exp \left(2 \varepsilon\left(N-N_{0}\right)\right) z_{N_{0}}, \quad N \geqslant N_{0} .
$$

Соединяя последнюю оценку с (4.17), выводим

$$
F_{m}(N) \leqslant C_{11} \exp \left(2 \varepsilon\left\{\frac{n(m-1)}{2(m+1)}+1\right\}\left(N-N_{0}\right)\right), \quad N \geqslant N_{0} .
$$

Таким образом, соотношение (0.33) установлено. 
Для монотонно неубывающей функции $f$, удовлетворяющей условию

$$
\sup \{f(z) \mid z \in[x-f(x), x+f(x)]\} \leqslant \omega f(x), \quad x \geqslant 1, \quad \omega \geqslant 1,
$$

справедливо неравенство

$$
\omega^{-1} f(r) \leqslant \rho_{*}(r) \leqslant f(r), \quad r \geqslant r_{0}=1+f(1) .
$$

В самом деле, в силу монотонного неубывания функции $f(x)$ справедливо равенство

$$
f\left(z_{*}\right)=r-z_{*}=\rho_{*}(r) .
$$

Согласно (4.18) имеем

$$
f(r)=\max _{\left[z_{*}-f\left(z_{*}\right), z_{*}+f\left(z_{*}\right)\right]} f(z) \leqslant \omega f\left(z_{*}\right),
$$

откуда следует левое неравенство (4.19).

Согласно (4.15), (4.19) функции $G_{m}(r), r_{1}(t)$ могут быть определены:

$$
\begin{gathered}
G_{m}(r)=f(r)\left(\int_{0}^{r} f^{n-1}(x) d x\right)^{(m-1) /(2(m+1))}, \quad r \geqslant r_{0} ; \\
\int_{1}^{r_{1}} \frac{d x}{f(x)}=\frac{t}{f^{2}\left(r_{1}\right)}, \quad t \geqslant t_{0} .
\end{gathered}
$$

ПримеР 1. Для функции $f(x)=x^{a}, 0 \leqslant a<1, x>0$, можно определить функции

$$
\begin{gathered}
r_{m}(t)=(\ln t)^{1 /(1-a)}, \quad t \geqslant t_{0}, \quad g_{m}(r)=r^{\chi}, \quad r \geqslant r_{0}, \quad m>1 \\
r_{1}(t)=t^{1 /(1+a)}, \quad t \geqslant t_{0} .
\end{gathered}
$$

Тогда оценки (0.42), (0.43) приобретают вид соответственно (0.44), (0.45).

ПримеР 2. Для функции $f(x)=e, 0<x<e, f(x)=x / \ln x, x \geqslant e$, находим

$$
\begin{aligned}
& r_{m}(t)=\exp \left(\varsigma(\ln t)^{1 / 2}\right), \\
& t \geqslant t_{0}, \quad \varsigma>0, \quad g(r)=r^{\sigma+1 / 2}(\ln r)^{-\sigma}, \quad r \geqslant r_{0}, \quad m>1 ; \\
& r_{1}(t)=t^{1 / 2}, \quad t \geqslant t_{0} .
\end{aligned}
$$

При этом оценки (0.42), (0.43) принимают вид соответственно (0.46), (0.47).

Авторы выражают искреннюю благодарность Ф. Х. Мукминову и А. К. Гущину за обсуждение результатов работы и полезные замечания.

\section{Список литературы}

[1] А. К. Гущин, "Об оценках решений краевых задач для параболического уравнения второго порядка", Краевые задачи для дифференциальных уравнений - 3 , Тр. МИАН, 126, Наука, М., 1973, 5-45; англ. пер.: A. K. Gushchin, "On estimates of the solutions of boundary value problems for a parabolic equation of second order", Proc. Steklov Inst. Math., 126 (1975), 1-46. 
[2] А.К. Гущин, "Стабилизация решений второй краевой задачи для параболического уравнения второго порядка", Матем. сб., 101(143):4(12) (1976), 459-499; англ. пер.: А. K. Guščin, "Stabilization of the solutions of the second boundary value problem for a second order parabolic equation", Math. USSR-Sb., 30:4 (1976), 403-440.

[3] Л.М.Кожевникова, Ф.Х. Мукминов, "Оценки скорости стабилизации при $t \rightarrow \infty$ решения первой смешанной задачи для квазилинейной системы параболических уравнений второго порядка", Матем. сб., 191:2 (2000), 91-131; англ. пер.: L. M. Kozhevnikova, F. Kh. Mukminov, "Estimates of the stabilization rate as $t \rightarrow \infty$ of solutions of the first mixed problem for a quasilinear system of second-order parabolic equations", Sb. Math., 191:2 (2000), 235-273.

[4] Л. М. Кожевникова, "Стабилизация решения первой смешанной задачи для эволюционного квазиэллиптического уравнения", Матем. сб., 196:7 (2005), 67-100; англ. пер.: L. M. Kozhevnikova, "Stabilization of a solution of the first mixed problem for a quasi-elliptic evolution equation", Sb. Math., 196:7 (2005), 999-1032.

[5] А. Ф. Тедеев, "Стабилизация решений первой смешанной задачи для квазилинейного параболического уравнения высокого порядка", Дифферени. уравения, 25:3 (1989), 491-498; англ. пер.: А. F. Tedeev, "Stabilization of solutions of a first mixed boundary-value problem for a high-order quasilinear parabolic equation", Differential Equations, 25:3 (1989), 346-352.

[6] Ф.Х. Мукминов, "Об убывании нормы решения смешанной задачи для параболического уравнения высокого порядка", Дифферени. уравнения, 23:10 (1987), 1772-1780; англ. пер.: F. H. Mukminov, "Decrease with time of the norm of a solution of a mixed problem for a high-order parabolic equation", Differential Equations, 23:10 (1987), 1215-1220.

[7] А.Ф. Тедеев, "Стабилизация решений начально-краевых задач для квазилинейных параболических уравнений", Укр. матем. журн., 44:10 (1992), 1441-1450; англ. пер.: A.F. Tedeev, "Stabilization of the solutions of initial-boundary value problems for quasilinear parabolic equations", Ukrainian Math. J., 44:10 (1992), 1325-1334.

[8] N.D. Alikakos, R. Rostamian, "Gradient estimates for degenerate diffusion equations. II", Proc. Roy. Soc. Edinburgh Sect. A, 91:3-4 (1982), 335-346.

[9] А. Ф. Тедеев, “Оценки скорости стабилизации при $t \rightarrow \infty$ решения второй смешанной задачи для квазилинейного параболического уравнения второго порядка", Дифферени. уравнения, 27:10 (1991), 1795-1806; англ. пер.: А. F. Tedeev, "Estimation of the stabilization rate for $t \rightarrow \infty$ of a solution of a second mixed problem for a second-order quasilinear parabolic equation", Differential Equations, 27:10 (1991), 1274-1283.

[10] А.Ф. Тедеев, "Стабилизация решения третьей смешанной задачи для квазилинейных параболических уравнений второго порядка в нецилиндрической области", Изв. вузов. Матем., 1 (1991), 63-73; англ. пер.: А.F. Tedeev, "The stabilization of solution of the third mixed problem for quasilinear parabolic equations of second order in the noncylindric domain", Soviet Math. (Iz. VUZ), 35:1 (1991), 75-87.

[11] С.П. Дегтярев, А.Ф. Тедеев, " $L_{1}-L_{\infty}$ оценки решения задачи Коши для анизотропного вырождающегося параболического уравнения с двойной нелинейностью и растущими начальными данными", Матем. сб., 198:5 (2007), 45-66; англ. пер.: S.P. Degtyarev, A.F. Tedeev, " $L_{1}-L_{\infty}$ estimates of solutions of the Cauchy problem for an anisotropic degenerate parabolic equation with double non-linearity and growing initial data", Sb. Math., 198:5 (2007), 639-660.

[12] А. В. Мартыненко, "Оценка максимума решений задачи Коши для вырождающегося параболического уравнения с неоднородной плотностью и источником", Труды ИПММ, 16, ИПММ НАН Украины, Донецк, 2008, 136-150. 
[13] Ф.Х. Мукминов, "Стабилизация решений первой смешанной задачи для параболического уравнения второго порядка", Матем. сб., 111(153):4 (1980), 503-521; англ. пер.: F. H. Mukminov, "Stabilization of solutions of the first mixed problem for a parabolic equation of second order", Math. USSR-Sb., 39:4 (1981), 449-467.

[14] Л.М. Кожевникова, Ф.Х. Мукминов, "Убывание решения первой смешанной задачи для параболического уравнения высокого порядка с младшими членами", Фундамент. и прикл. матем., 12:4 (2006), 113-132; англ. пер.: L. M. Kozhevnikova, F. H. Mukminov, "Decay of solutions of the first mixed problem for a high-order parabolic equation with minor terms", J. Math. Sci. (N. Y.), 150:5 (2008), 2369-2383.

[15] Л.М.Кожевникова, "Поведение на бесконечности решений псевдодифференциальных эллиптических уравнений в неограниченных областях", Матем. сб., 199:8 (2008), 61-94; англ. пер.: L. M. Kozhevnikova, "Behaviour at infinity of solutions of pseudodifferential elliptic equations in unbounded domains", Sb. Math., 199:8 (2008), 1169-1200.

[16] Л.М. Кожевникова, "Стабилизация решений псевдодифференциальных параболических уравнений в неограниченных областях", Изв. РАН. Сер. матем., 74:2 (2010), 109-130; англ. пер.: L. M. Kozhevnikova, "Stabilization of solutions of pseudo-differential parabolic equations in unbounded domains", Izv. Math., 74:2 (2010), 325-345.

[17] О.А. Олейник, Г. А. Иосифьян, "О единственности решения смешанной задачи для уравнений теории упругости в неограниченной области", УМH, 31:5 (1976), $247-248$.

[18] О.А. Ладыженская, В.А. Солонников, Н.Н. Уральцева, Линейнъе и квазилинейные уравнения параболического типа, Наука, М., 1967; англ. пер.: O. A. Ladyženskaja, V.A. Solonnikov, N. N. Ural'ceva, Linear and quasilinear equations of parabolic type, Transl. Math. Monogr., 23, Amer. Math. Soc., Providence, RI, 1968.

\section{P. X. Каримов (R. Kh. Karimov)}

Институт прикладных исследований, г. Стерлитамак

E-mail: ruslan7k7@mail.ru

\section{Л. М. Кожевникова (L. M. Kozhevnikova)}

Стерлитамакская государственная педагогическая

академия им. 3. Биишевой

E-mail: kosul@mail.ru
Поступила в редакцию

16.07.2009 и 08.04.2010 